Document downloaded from:

http://hdl.handle.net/10251/101904

This paper must be cited as:

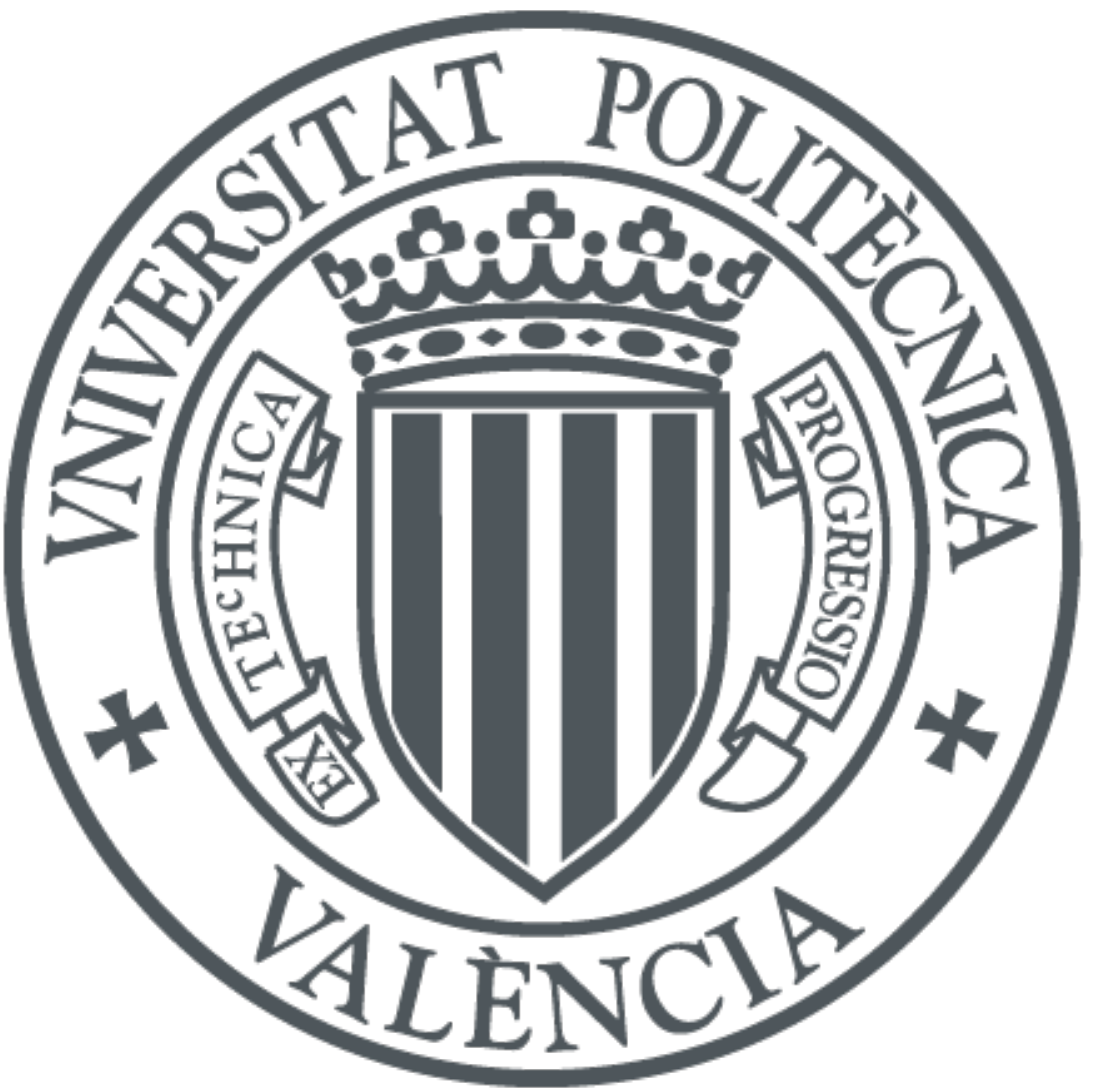

The final publication is available at

https://doi.org/10.1016/j.engstruct.2017.09.045

Copyright Elsevier

Additional Information 


\title{
A form-finding method based on the geometrically exact rod model for bending-active structures
}

\author{
J. Bessini*, C.Lázaro, S. Monleón \\ Departamento de Mecánica de los Medios Continuos y Teoría de Estructuras \\ Universitat Politècnica de València \\ Camino de Vera s/n, 46022, Valencia, Spain
}

This work is licensed under a Creative Commons "AttributionNonCommercial-NoDerivatives 4.0 International" license.

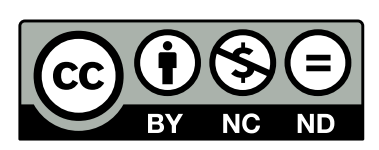

\begin{abstract}
In the field of bending-active structures, the complexity of finding beforehand the equilibrium configuration and the non-linearity of the structural response are main issues during the conceptual phase. The use of tools based on classical form-finding procedures as dynamic relaxation is the main trend today; different mechanical models with 3, 4 or 6 degrees of freedom have been implemented for modelling the bending effect. However, there is a well-established class of mechanical models which has been specifically designed to reproduce the behaviour of very flexible structures and has not been used so far in form-finding of bending-active structures. These are derived from the so-called geometrically exact (or Reissner-Simo) beam theory, and they are able to treat arbitrarily large rotations and displacements. In this paper, we present the development of a form-finding tool based on Reissner-Simo's theory and the dynamic relaxation method, in order to find the static equilibrium of the system. The choice of form-finding parameters as the target curve length and the kinematic constraints at beam ends will determine the shape of the final structure in the 'design-oriented' process. Several numerical examples on a range of structures are tested to validate the formulation.
\end{abstract}

\section{Introduction}

\subsection{Motivation and background}

Nowadays, modern architecture is focused on the search of efficient uses of technological and sustainable materials, high-tech concept-design-erection processes and the possibility to produce lightweight solutions with minimal impact and maximum elegance in shape. These ideas are reflected on bending-active structures, because they are assembled using slender members bent into attractive lightweight and shaped configurations. The term 'active-bending' refers to a category of structures in which bending is used in the process of shape configuration, obtaining structural schemes from elastic members such as beams or rods, which initially are straight and unstressed. However, the number of realizations is limited. The Mannheim Multihalle designed by Frei Otto,

*juabesmu@mes.upv.es 
which is a pioneering modern application of the bending active principle [1]; the Ephemeral Cathedral of Creteil (Paris, France) by du Peloux et al., gridshell structure made of composite materials [2]; the temporary ICD/ITKE Research pavilion made of plywood at the University of Stuttgart and the permanent textile umbrella for Marrakech [3] are some of the built examples.

Due to the non-linearity of the structural response of the active members, the simulation of the process of deformation of bending-active elements is one of the main difficulties during the conceptual phase. Moreover, it is often not possible to obtain beforehand the equilibrium description of the system; its shape has to be determined as a result of a tight interaction between form, forces, material properties and boundary conditions [4]. Form-finding methodologies such as finite element models with non-linear virtual links that are shortened to reach the final form [5], and dynamic relaxation methods based on beam models with 3 to 6 DoFs have been proposed by several authors [6, 7, 8, 9, 10, 11, 12, 13, 14.

Despite the availability of different mechanical models to describe the kinematics of flexible members, most formulations covered in the literature [7, 9, 8, 10, 11, 12, 13, 14] are based on Kirchhoff's theory [15], in which cross-sections remain almost normal to the centreline of the rod and almost planar, and seems adequate to handle the specificity of the mechanical problems posed by bending-active structures. However, among those mathematical models handling large rotations and displacements, the so called geometrically exact rod model or Reissner-Simo model has been specifically designed to reproduce the behaviour of very flexible structures.

Reissner [16] and Simo [17] developed a non-linear theory for the deformation of flexible rods that can be considered as a special case of Cosserat's theory, in which the director vectors attached to material fibres in a cross-section are constrained to remain inextensible and orthogonal, but not necessarily normal to the deformed centreline tangents. This theory can be also interpreted as a non-linear version of Timoshenko's theory for shear deformable beams and offers the conceptual simplicity of the kinematic assumption together with the power of a direct theory for solving nonlinear problems.

The assumptions of the geometrically exact model can be summarized as follows: cross-sections remain plane without changes of shape or size, although they may experience finite rotations as a rigid-body; warping effects are not modelled; the centreline can undergo extension, and shear deformations are allowed. Simo in 1991 [18, extended his previous work incorporating shear and torsion-warping deformation into the geometrically exact rod model. The consideration of warping would indeed allow to handle thin-walled open cross-sections and the authors hope to extend their work in this line. Hence, taking into account the potential of the geometrically exact rod model to handle arbitrarily large rotations and centreline displacements without limitations of magnitude, the authors present in this work a form-finding methodology for bending-active structures based on this mechanical model.

\subsection{Recent trends in form finding methods for bending active structures}

Traditionally, finite element models have been used for the load analysis and the simulation of the construction process of structures. In the case of evolutive processes and non-linear response, implicit resolution methods are often used to find equilibrium solutions. However, in the so called 'design-oriented' problems, where the main unknown is the geometrical configuration and the determination of deformations and stresses is not the main goal, the use of explicit methods has been reported by some authors [19, 13] as more advantageous for those cases in which prescribed conditions are far from the equilibrium geometry. As a starting point in the conceptual phase, physical models provide good geometrical approximations (Fig. 1), however, the initial pre-stress induced by the form-change is unknown. In this case, the development of numerical tools based on explicit methods as dynamic relaxation is the main trend today.

Dynamic relaxation provides a straightforward way to obtain the static equilibrium of the structure from a given arbitrary and inaccurate initial configuration. Moreover, the possibility of using 
fictitious stiffness, massess and time steps contributes to the fast convergence of the numerical solution. Several approaches for this method have been suggested over the years, being reduced models with only three translational DoFs the most used. Douthe et al. [8], focusing on the previous work done by Adriaenssens and Barnes [7 proposed to calculate the magnitude of bending moments through estimates of curvatures obtained from the positions of groups of three consecutive nodes. Bending moments are transformed into equivalent forces, acting on the actual geometry of the beam. This model is valid for initially straight beams with axisymmetric cross-section. The problem of torsion and bending for dynamic relaxation modelling of spatial curved beams was approached by Barnes et al. [9] few years later, extending Douthe's work; their assumption accounts for both torsion and transverse (out of plane) bending effects; this model is valid in cases where cross-sections are isotropic.

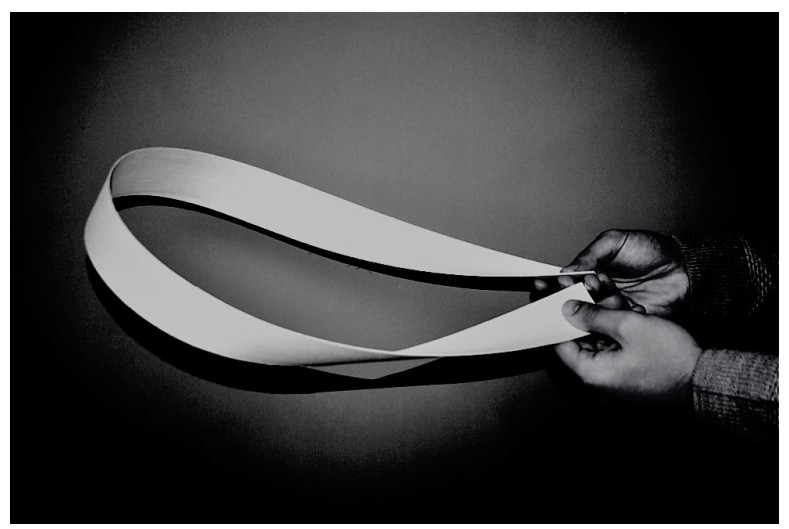

Figure 1: A physical model subject to bending and torsion effects. (Bending of developable surfaces has been studied in [20].)

The first proposal to handle the problem of slender beams in large displacements using dynamic relaxation was introduced by Wakefield in 1980 [21; he implemented a formulation taking into account 6 DoFs. As reported by Adriaenssens [22, Williams developed a more precise resolution scheme, assuming local reference frames at nodes and cubic shape functions to simulate the stiffness of the rod. Few years ago and based on Williams's work, D'Amico et al. [10 built a numerical framework aimed to the design of gridshells. Senatore and Piker [12] used a corotational approach to compute the resultant field of displacements and the effect of large deformations. The use of 6 DoFs per node has the advantage of providing a complete description of the mechanical response. However, Adriaenssens and Barnes mention in reference [7] that the coupling between translational and rotational DoFs can cause conditioning problems in explicit methods.

In recent years, the study of thin flexible rods has also been developed in the field of computer animation and games since this industry requires fast simulation techniques. For example, Bergou et al. [23] present a discrete treatment based on the study of adapted framed curves and the topological concepts of parallel transport and holonomy. In contrast to typical simulation techniques relying on a kinematic description, they treat the centreline as dynamic and the material frame as quasi-static defined by its angular deviation from the natural Bishop frames, that are torsionfree trihedrons related to Frenet's trihedrons by a rotation around the tangent vector. Thereby, solutions are formulated with a minimal number of degrees of freedom: the explicit description of the centreline of the rod (three DoFs) and the orientation of material frame using a scalar variable (one additional $\mathrm{DoF}$ ). The authors claim that using this procedure, numerical instabilities are avoided in the dynamic relaxation algorithm. Based on these developments in the field of computer animation, du Peloux et al. [11, D'Amico et al. 13] and Lefevre et al. 14 have recently presented alternative approaches to model elastic rods with 4 -DoFs. These formulations allow to 
deal with general cases of structures with anisotropic cross-sections and torsional stiffness effects by means of Bishop frames. In the beam model developed by D'Amico et al., the definition of boundary conditions is not straightforward and pre-processing is necessary, defining extra-nodes or constraints.

Different strategies have been carried out to perform dynamic relaxation in order to obtain accurate and fast simulations, having in common Kirchhoff's model as kinematic model. However, the authors propose the use of a mechanical model specifically designed to deal with large displacements and rotations. An additional advantage of the model is that it is appropiate for modelling structures in which shear deformation is not negligible. In the realm of bending-active structures, it could be applied to structures formed by two o more layers of laths, considering them as unitary structural elements with large shear deformations (e.g. the Mannheim Multihalle). Therefore, this work presents a form-finding method based on the geometrically exact rod model with 6 DoFs, aimed to achieve powerful numerical solutions thanks to the conceptual simplicity of the underlying mathematical theory and its specific design for flexible structures.

\subsection{Assumptions and starting points}

The proposed form-finding method is based on the following considerations:

- Explicit solution search using dynamic relaxation.

- The geometrically exact rod model as underlying mechanical model.

- Translations and rotations are updated by means of 6 DoFs per node.

- Anisotropic cross-sections can be modelled. Thin-walled open cross-sections are precluded.

- Initial specifications of the geometry do not need to be close to the equilibrium solution.

- Boundary conditions are defined by nodal positions and cross-section orientations (optionally) of the beam ends. Additional numerical constraints or nodes are unnecessary.

\subsection{Outline of the paper}

The outline of the paper is as follows: In section 2, the principles of the dynamic relaxation method as well as the fundamentals of the geometrically exact model are reviewed. Furthermore, the description of the numerical framework proposed by the authors is detailed. Numerical examples showing the ability of the form-finding method to reproduce the behaviour of active members are included in section 3 .

\section{Dynamic relaxation using the geometrically exact model}

\subsection{The geometrically exact rod model}

The geometrically exact rod model was formulated by Simo [17] starting from the work of Reissner [16. It allows to reproduce arbitrarily large displacements and rotations of rods using an exact kinematic description of finite rotations of cross-sections. In this section, a brief summary of the fundamentals of Reissner-Simo theory is given. The notation we use is based, although not the same, as the one introduced by Simo. It follows more closely the one used by Ritto-Corrêa and Camotim in their summarizing reference [24].

\subsubsection{Basic kinematics}

A fixed reference system $\{x, y, z\}$ in space is chosen. Three configurations are considered (Fig. 22): a) The reference (ideal) configuration, in which the rod is straight, the line of centroids is aligned with the $x$-axis, and cross-sections are oriented in such way that principal axes of inertia are parallel 
to $y$ and $z$-axes. Unit vectors $\mathbf{A}_{2}$ and $\mathbf{A}_{3}$ are attached to the principal axes and $\mathbf{A}_{1}$ is normal to the cross-sections; they form an orthogonal system. $S$ is the (arc-)length coordinate along the centreline in the reference configuration. $X^{2}$ and $X^{3}$ are coordinates in each cross-section. b) The initial (undeformed) configuration: the rod centreline can be a spatial curve. Cross-sections are normal to the curve and are defined by $\mathbf{a}_{01}, \mathbf{a}_{02}, \mathbf{a}_{03}$, the unit directors in the underformed configuration, being $\mathbf{a}_{01}$ tangent to the curve. These directors are the result of a rigid-body rotation of $\mathbf{A}_{1}, \mathbf{A}_{2}, \mathbf{A}_{3}$. c) The actual (deformed) configuration at an instant $t$ : during the deformation every cross-section is assumed to undergo a rigid-body motion. Directors $\mathbf{a}_{1}, \mathbf{a}_{2}, \mathbf{a}_{3}$ remain unitary and orthogonal, but $\mathbf{a}_{1}$ is not necessarily tangent to the centreline. If the components with respect to the fixed space frame of $\mathbf{a}_{1}, \mathbf{a}_{2}$ and $\mathbf{a}_{3}$ are written in columns, they form an orthogonal matrix $\boldsymbol{\Lambda}$ of determinant 1, which is only a function of $S$ and the time $t$. The position vector of a material point can be expressed in terms of its relative location into the section $\mathbf{r}^{*}$, the position of the centroid of the section $\mathrm{x}$ and the rotation of the cross-section $\boldsymbol{\Lambda}$ as follows:

$$
\begin{aligned}
& \mathbf{x}^{*}\left(S, X^{2}, X^{3}, t\right)=\mathbf{x}(S, t)+\mathbf{r}^{*}\left(S, X^{2}, X^{3}, t\right) \\
& \mathbf{r}^{*}\left(S, X^{2}, X^{3}, t\right)=\boldsymbol{\Lambda}(S, t) \mathbf{R}^{*}\left(X^{2}, X^{3}\right)
\end{aligned}
$$

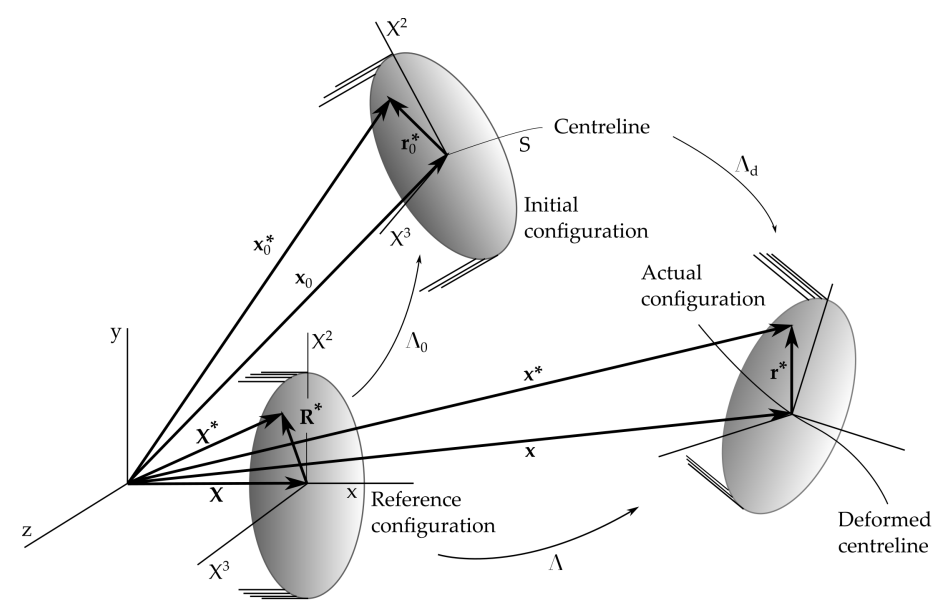

Figure 2: Kinematics of Reissner-Simo's model [25].

Section points rotate from an ideal reference configuration (described by $\boldsymbol{R}^{*}$ ) to the deformed (actual) configuration. $\mathbf{x}(S, t)$ and $\boldsymbol{\Lambda}(S, t)$ are the configuration functions of the geometrically exact model. The 1D deformation gradient can be written as [25]:

$$
\partial \mathbf{x}^{*} / \partial S=\mathbf{x}^{\prime}+\boldsymbol{\Lambda}^{\prime} \Lambda^{T} \mathbf{r}^{*}=\gamma+\hat{\kappa} \mathbf{r}^{*}
$$

The hat over a variable denotes a skew-symmetric matrix. The same variable with no hat refers to its axial vector. Capital letters refer to material variables (variables expressed in the system of reference attached to the cross-sections). Lowercase letters refer to spatial variables (variables expressed in the fixed reference system $\{x, y, z\}$ ). Transformations between spatial and material forms is performed through the action of $\boldsymbol{\Lambda}$. Given a vector $\mathbf{v}$, the operation $\mathbf{v}=\boldsymbol{\Lambda} \mathbf{V}$ is called push-forward, and the operation $\mathbf{V}=\boldsymbol{\Lambda}^{T} \mathbf{v}$ is called pull-back.

$\gamma$ and $\kappa$ are the generalized deformations expressed in spatial form. Their counterparts in 
material form, $\boldsymbol{\Gamma}$ and $\mathbf{K}$, allow to define the physical deformations $\boldsymbol{\Gamma}_{d}$ and $\mathbf{K}_{d}$ as:

$$
\begin{aligned}
\boldsymbol{\Gamma}_{d} & =\boldsymbol{\Gamma}-\boldsymbol{\Gamma}_{0} \\
\mathbf{K}_{d} & =\mathbf{K}-\mathbf{K}_{0}
\end{aligned}
$$

$\hat{\mathbf{K}}_{0}=\boldsymbol{\Lambda}_{0}^{T} \boldsymbol{\Lambda}_{0}^{\prime}$ represents the curvature and twist of the initial centreline, and $\boldsymbol{\Gamma}_{0}$ is the material form of the tangent vector to the initial centreline. $\boldsymbol{\Gamma}_{0}$ is precisely the vector $\mathbf{A}_{1}$ of the section frame in the reference configuration, with components $\{1,0,0\}$. Therefore:

$$
\boldsymbol{\Gamma}_{d}=\boldsymbol{\Gamma}-\mathbf{A}_{1}=\left\{\Gamma_{1}-1, \Gamma_{2}, \Gamma_{3}\right\}
$$

The first component of $\boldsymbol{\Gamma}_{d}$ is a measure of the elongation of the centreline and the other two components are the distortions along the axes of the cross-section. It is worth remarking that $\mathbf{K}_{0}$ $=\mathbf{0}$ when the rod is initially straight, something usual in the initial definition of geometries in form-finding problems.

\subsubsection{Variation of the generalised deformations}

The variation of the configuration can be computed as:

$$
\delta \mathbf{x}^{*}=\delta \mathbf{x}+\delta \boldsymbol{\Lambda}^{\prime} \boldsymbol{\Lambda}^{T} \mathbf{r}^{*}=\delta \mathbf{x}+\delta \hat{\boldsymbol{\omega}} \mathbf{r}^{*}
$$

$\delta \boldsymbol{\omega}$, the axial vector of $\delta \hat{\boldsymbol{\omega}}$, is referred to as the incremental rotation or spin. Because $\boldsymbol{\gamma}$ and $\boldsymbol{\kappa}$ are mathematical objects related to the (moving) cross-section, their variation requires a special definition: given a vector $\mathbf{v}$, the corotational variation $\stackrel{\nabla}{\delta} \mathbf{v}$ is defined as the variation from the point of view of an observer located at the section reference system as:

$$
\stackrel{\nabla}{\delta} \mathbf{v}=\boldsymbol{\Lambda} \delta\left(\boldsymbol{\Lambda}^{T} \mathbf{v}\right)=\delta \mathbf{v}-\delta \boldsymbol{\omega} \times \mathbf{v}
$$

therefore, the co-rotational variations of the generalized deformations are:

$$
\stackrel{\nabla}{\delta} \boldsymbol{\gamma}=\delta \mathbf{x}^{\prime}+\boldsymbol{\gamma} \times \delta \boldsymbol{\omega} \quad \text { and } \quad \stackrel{\nabla}{\delta} \boldsymbol{\kappa}=(\delta \boldsymbol{\omega})^{\prime}
$$

\subsubsection{Equations of static equilibrium}

First, the expression of the internal virtual work is manipulated. Introducing the Eqs. (7) in the term corresponding to the internal forces $\boldsymbol{n}$ and moments $\boldsymbol{m}$ in the spatial description, the expression reads:

$$
\delta W_{i n t}=\int_{S_{i}}^{S_{j}}(\boldsymbol{n} \cdot \stackrel{\nabla}{\delta} \boldsymbol{\gamma}+\boldsymbol{m} \cdot \stackrel{\nabla}{\delta} \boldsymbol{\kappa}) d S
$$

and integrating by parts:

$$
\begin{gathered}
\int_{S_{i}}^{S_{j}}(-\boldsymbol{n}) \cdot \delta \boldsymbol{x} d S+\int_{S_{i}}^{S_{j}}\left(-\boldsymbol{m}^{\prime}+\boldsymbol{n} \times \gamma\right) \cdot \delta \boldsymbol{\omega} d S \\
+[\boldsymbol{n} \cdot \delta \boldsymbol{x}]_{S_{i}}^{S_{j}}+[\boldsymbol{m} \cdot \delta \boldsymbol{\omega}]_{S_{i}}^{S_{j}}
\end{gathered}
$$

The virtual work of the external forces $\boldsymbol{q}_{n}$ and $\boldsymbol{q}_{m}$ is:

$$
\delta W_{e x t}=\int_{S_{i}}^{S_{j}}\left(\boldsymbol{q}_{n} \cdot \delta \boldsymbol{x}+\boldsymbol{q}_{m} \cdot \delta \boldsymbol{\omega}\right) d S
$$


Static equilibrium is equivalent to the equality of internal and external virtual work $\delta W_{\text {int }}=\delta W_{\text {ext }}$ for any compatible set of virtual movements of the system. Therefore, the spatial form of the equations of static equilibrium are:

$$
\begin{aligned}
\boldsymbol{n}^{\prime}+\boldsymbol{q}_{n} & =\mathbf{0} \\
\boldsymbol{m}^{\prime}+\boldsymbol{\gamma} \times \boldsymbol{n}+\boldsymbol{q}_{m} & =\mathbf{0}
\end{aligned}
$$

with the boundary conditions at the start:

$$
\begin{array}{r}
{\left[\boldsymbol{n}\left(S_{i}\right)+\boldsymbol{n}_{i}\right] \cdot \delta \boldsymbol{x}\left(S_{i}\right)=0} \\
{\left[\boldsymbol{m}\left(S_{i}\right)+\boldsymbol{m}_{i}\right] \cdot \delta \boldsymbol{\omega}\left(S_{i}\right)=0}
\end{array}
$$

and the boundary conditions at the end:

$$
\begin{array}{r}
{\left[\boldsymbol{n}\left(S_{j}\right)+\boldsymbol{n}_{j}\right] \cdot \delta \boldsymbol{x}\left(S_{j}\right)=0} \\
{\left[\boldsymbol{m}\left(S_{j}\right)+\boldsymbol{m}_{j}\right] \cdot \delta \boldsymbol{\omega}\left(S_{j}\right)=0}
\end{array}
$$

On the other hand, the internal virtual work in the spatial configuration can be expressed in matrix form as:

$$
\begin{gathered}
\delta W_{\text {int }}=\int_{S_{i}}^{S_{j}} \stackrel{\nabla}{\delta} \cdot \boldsymbol{f} d S=\int_{S_{i}}^{S_{j}}(\stackrel{\nabla}{\delta} \cdot \boldsymbol{n}+\stackrel{\nabla}{\delta} \boldsymbol{\kappa} \cdot \boldsymbol{m}) d S \\
=\int_{S_{i}}^{S_{j}}\left\{\delta \boldsymbol{x}^{T} \delta \boldsymbol{\omega}^{T} \delta \boldsymbol{x}^{\prime T} \delta \boldsymbol{\omega}^{\prime T}\right\}\left[\begin{array}{cc}
\mathbf{0} & \mathbf{0} \\
-\hat{\boldsymbol{\gamma}} & \mathbf{0} \\
\mathbf{1} & \mathbf{0} \\
\mathbf{0} & \mathbf{1}
\end{array}\right]\left\{\begin{array}{c}
\boldsymbol{n} \\
\boldsymbol{m}
\end{array}\right\} d S
\end{gathered}
$$

\subsubsection{Equations of dynamic equilibrium}

To account for dynamic effects, Simo [17] introduced the equations of motion expressed in terms of forces and moments:

$$
\begin{aligned}
\boldsymbol{n}^{\prime}+\boldsymbol{q}_{n} & =\mathrm{m} \boldsymbol{a} \\
\boldsymbol{m}^{\prime}+\gamma \times \boldsymbol{n}+\boldsymbol{q}_{m} & =\AA \dot{\boldsymbol{\omega}}+\boldsymbol{\omega} \times \AA \boldsymbol{\omega}
\end{aligned}
$$

where $m$ is the mass matrix, 0 the inertia tensor, $\boldsymbol{a}$ is the translational acceleration vector, $\boldsymbol{\omega}$ is the angular velocity vector and $\dot{\boldsymbol{\omega}}$ the angular acceleration vector, all of them expressed in spatial form.

\subsubsection{Constitutive equations}

The material form of the constitutive equations relates the static variables $\boldsymbol{N}$ and $\boldsymbol{M}$ with the physical deformations of the model $\boldsymbol{\Gamma}_{d}$ and $\boldsymbol{K}_{d}$ as follows:

$$
\left\{\begin{array}{l}
N \\
M
\end{array}\right\}=\left[\begin{array}{cc}
C_{\Gamma} & \mathbf{0} \\
\mathbf{0} & C_{K}
\end{array}\right]\left\{\begin{array}{l}
\Gamma_{d} \\
\boldsymbol{K}_{d}
\end{array}\right\}
$$

Assuming that the centroid and the centre of torsion of the cross-section coincide, $\boldsymbol{C}_{\Gamma}$ and $\boldsymbol{C}_{K}$ are:

$$
\begin{aligned}
C_{\Gamma} & =\left[\begin{array}{ccc}
E A & 0 & 0 \\
0 & G A_{Q 2} & 0 \\
0 & 0 & G A_{Q 3}
\end{array}\right] \\
C_{K} & =\left[\begin{array}{ccc}
G J & 0 & 0 \\
0 & E I_{2} & 0 \\
0 & 0 & E I_{3}
\end{array}\right]
\end{aligned}
$$


The spatial description of the internal forces is obtained pushing-forward the corresponding material variables:

$$
\left\{\begin{array}{c}
n \\
m
\end{array}\right\}=\left[\begin{array}{cc}
\Lambda & 0 \\
0 & \Lambda
\end{array}\right]\left\{\begin{array}{l}
N \\
M
\end{array}\right\}
$$

\subsection{The dynamic relaxation method}

The dynamic relaxation method (DR) was introduced in the 60s by Day [26] and Otter [27. It was developed to determine the equilibrium configuration of a system considering it as a fictitious dynamic system; the initial specification of the geometry can be far from the equilibrum solution. The equilibrium configuration is obtained tracing step-by-step the motion of each node of the structure for small time increments $\Delta t$ using Newton's second law, until the equlibrium state is reached [19]. In order to achieve rapid convergence, several approaches can be considered, for example the use of viscous damping. When the structure has large motions, additional damping strategies (as kinetic damping) must be implemented. Being an explicit method, DR does not require assembling any stiffness matrix and it is quick to build the system, providing powerful and reliable results for non-linear structural problems.

\subsection{Fundamentals of the method}

This section shows the fundamentals of the implementation of the geometrical exact rod model into the dynamic relaxation method.

An elastic rod is modelled by a discrete set of nodes $\boldsymbol{x}_{i}$, which are referred to the fixed Cartesian coordinate system (global reference system $\{x, y, z\}$ ) These nodes are linked in pairs, being at the same time, the ends of beam elements that constitute the whole rod.

A local reference frame is associated to each node. The unit vectors of the frame define the orientation of corresponding cross-section. At the start of the process, nodal frames can be arbitrarily chosen at free nodes.

In addition to kinematic constraints, the parameter $L_{0}$, target length of the curve in the equilibrium state, must be prescribed. The sum of distances between nodes is near the prescribed target length of the elements when the state of equilibrium is reached.

\subsubsection{Application of D'Alembert's principle}

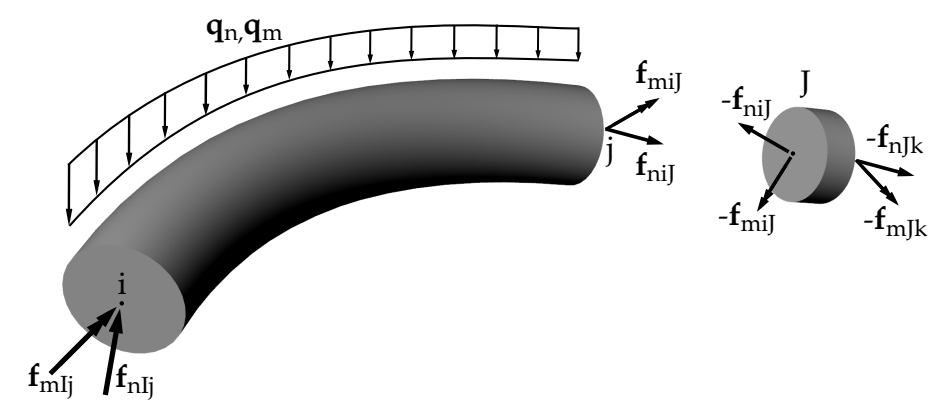

Figure 3: Scheme of forces and moments acting on the element ij and the node $J$

The fundamental of the method is based on D'Alembert's principle. Dynamic equilibrium is expressed using Eqs. $15 \mathrm{a}$ and $15 \mathrm{~b}$, where out of balance forces $\tilde{\boldsymbol{f}}_{n_{J}}$ and out of balance moments $\tilde{\boldsymbol{f}}_{m_{J}}$ at node $J$ are equal to the derivative of the linear momentum $\dot{\boldsymbol{p}}_{J}$ and the derivative of the 
angular momentum $\dot{\boldsymbol{h}}_{J}$ acting on this node:

$$
\begin{aligned}
& \tilde{\boldsymbol{f}}_{n_{J}}=\dot{\boldsymbol{p}}_{J}=\mathrm{m}_{J} \boldsymbol{a}_{J} \\
& \tilde{\boldsymbol{f}}_{m_{J}}=\dot{\boldsymbol{h}}_{J}=\AA_{J} \dot{\boldsymbol{\omega}}_{J}+\boldsymbol{\omega}_{J} \times \AA_{J} \boldsymbol{\omega}_{J}
\end{aligned}
$$

On the other hand, the out of balance forces/moments are the resultant of the external loads and the internal forces/moments caused by elements attached to node $J$ :

$$
\begin{gathered}
\tilde{\boldsymbol{f}}_{n_{J}}=-\boldsymbol{f}_{n i J}-\boldsymbol{f}_{n_{J} k} \\
\tilde{\boldsymbol{f}}_{m_{J}}=-\boldsymbol{f}_{m i J}-\boldsymbol{f}_{m_{J} k}
\end{gathered}
$$

Evaluating iteratively the equations of the dynamic equilibrium at nodes, the update of the geometry is provided by the motion associated to each set of out of balance forces/moments. The state of equilibrium is reached when the structure comes to rest; the sum of all forces/moments is zero at each node.

Internal forces/moments at element ends are computed according to D'Alembert's principle: using the notation of Fig. 3 , the following equation holds for any consistent set of virtual displacements $\delta \boldsymbol{x}$ and spins $\delta \boldsymbol{\omega}$ :

$$
\begin{aligned}
& \int_{S_{i}}^{S_{j}}\left(-\boldsymbol{n}-\boldsymbol{q}_{n}+\mathrm{m} \boldsymbol{a}\right) \cdot \delta \boldsymbol{x} d S \\
& +\int_{S_{i}}^{S_{j}}\left(-\boldsymbol{m}^{\prime}+\boldsymbol{n} \times \boldsymbol{\gamma}-\boldsymbol{q}_{m}+\AA \dot{\boldsymbol{\omega}}+\boldsymbol{\omega} \times \AA \boldsymbol{\omega}\right) \cdot \delta \boldsymbol{\omega} d S \\
& +\left[\boldsymbol{n}_{I j}-\boldsymbol{f}_{n_{I j}}\right] \delta \boldsymbol{x}_{i}+\left[\boldsymbol{m}_{I j}-\boldsymbol{f}_{m_{I j}}\right] \delta \boldsymbol{\omega}_{i} \\
& +\left[\boldsymbol{n}_{i J}-\boldsymbol{f}_{n_{i J}}\right] \delta \boldsymbol{x}_{j}+\left[\boldsymbol{m}_{i J}-\boldsymbol{f}_{m_{i J}}\right] \delta \boldsymbol{\omega}_{j}=0
\end{aligned}
$$

Assuming that masses and inertias are lumped at the nodes, angular velocity and acceleration terms vanish from the integrals. Rewriting the boundary conditions in matrix form, we have:

$$
\begin{aligned}
\int_{S_{i}}^{S_{j}}(-\boldsymbol{n}) & \cdot \delta \boldsymbol{x} d S+\int_{S_{i}}^{S_{j}}\left(-\boldsymbol{m}^{\prime}+\boldsymbol{n} \times \boldsymbol{\gamma}\right) \cdot \delta \boldsymbol{\omega} d S \\
& -\int_{S_{i}}^{S_{j}}\left(\boldsymbol{q}_{n} \cdot \delta \boldsymbol{x}+\boldsymbol{q}_{m} \cdot \delta \boldsymbol{\omega}\right) d S \\
= & \left\{\delta \boldsymbol{x}_{i} \delta \boldsymbol{\omega}_{i} \delta \boldsymbol{x}_{j} \delta \boldsymbol{\omega}_{j}\right\}\left\{\begin{array}{c}
\boldsymbol{f}_{n I j} \\
\boldsymbol{f}_{m I j} \\
\boldsymbol{f}_{n i J} \\
\boldsymbol{f}_{m i J}
\end{array}\right\}
\end{aligned}
$$

\subsubsection{Determination of element end forces}

In Eq. 22, the first line is coincident with the expression of the internal virtual work in static equilibrium. Introducing a Lagrangian linear interpolation in Eq. (14), the contribution of each element to the internal virtual work can be discretized. Considering two-node beam elements and using an intrinsic coordinate $\xi$, which takes $\xi=-1$ when $S=S_{i}$ and $\xi=1$ when $S=S_{j}$, internal forces and moments can be evaluated at their ends as follows:

$$
\delta W_{i n t}=\delta \phi^{T} \int_{-1}^{1}\left[\begin{array}{ccccc}
\mathrm{N}_{0} \mathbf{1} & \mathbf{0} & \frac{1}{J} \mathrm{~N}_{0}^{\prime} \mathbf{1} & \mathbf{0} \\
\mathbf{0} & \mathrm{N}_{0} \mathbf{1} & \mathbf{0} & \frac{1}{J} \mathrm{~N}_{0}^{\prime} & \mathbf{1} \\
\mathrm{N}_{1} \mathbf{1} & \mathbf{0} & \frac{1}{J} \mathrm{~N}_{1}^{\prime} \mathbf{1} & \mathbf{0} \\
\mathbf{0} & \mathrm{N}_{1} \mathbf{1} & \mathbf{0} & \frac{1}{J} \mathrm{~N}_{1}^{\prime} & \mathbf{1}
\end{array}\right]\left[\begin{array}{cc}
\mathbf{0} & \mathbf{0} \\
-\hat{\gamma} & \mathbf{0} \\
\mathbf{1} & \mathbf{0} \\
\mathbf{0} & \mathbf{1}
\end{array}\right]\left\{\begin{array}{c}
\boldsymbol{n} \\
\boldsymbol{m}
\end{array}\right\} J d \xi
$$


where $\mathrm{N}_{0}$ and $\mathrm{N}_{1}$ are the shape-functions for two-node beam elements based on Lagrange polynomials and $\mathrm{N}_{0}^{\prime}$ and $\mathrm{N}_{1}^{\prime}$ their derivatives:

$$
\mathrm{N}_{0}=\frac{1}{2}(1-\xi) \quad \text { and } \quad \mathrm{N}_{1}=\frac{1}{2}(1+\xi)
$$

$\delta \phi^{T}$ is $\left\{\delta \boldsymbol{x}_{i} \delta \boldsymbol{\omega}_{i} \delta \boldsymbol{x}_{j} \delta \boldsymbol{\omega}_{j}\right\}$ and $J$ is the Jacobian of the transformation that relates $S$ and $\xi$, where $\mathrm{S} \in\left[0, L_{e}\right]$. (Not to be confused with the node nomenclature.):

$$
J(\xi)=\frac{d S}{d \xi}=\left[\begin{array}{ll}
\mathrm{N}_{0}^{\prime}(\xi) & \mathrm{N}_{1}^{\prime}(\xi)
\end{array}\right]\left\{\begin{array}{c}
\boldsymbol{S}_{\boldsymbol{i}} \\
\boldsymbol{S}_{\boldsymbol{j}}
\end{array}\right\}=\frac{L_{e}}{2}
$$

Finally, the out of balance forces/moments (disregarding external forces) are obtained computing the previous equation in the elements connected to node $J$ :

$$
\begin{aligned}
& \left\{\begin{array}{c}
\tilde{\boldsymbol{f}}_{n_{J}} \\
\tilde{\boldsymbol{f}}_{m_{J}}
\end{array}\right\}=-\left\{\begin{array}{c}
\boldsymbol{f}_{n_{i J}} \\
\boldsymbol{f}_{m_{i J}}
\end{array}\right\}-\left\{\begin{array}{c}
\boldsymbol{f}_{n_{J k}} \\
\boldsymbol{f}_{m_{J k}}
\end{array}\right\} \\
& =-\int_{-1}^{1}\left[\begin{array}{cccc}
\mathrm{N}_{1} \mathbf{1} & \mathbf{0} & \frac{1}{J} \mathrm{~N}_{1}^{\prime} \mathbf{1} & \mathbf{0} \\
\mathbf{0} & \mathrm{N}_{1} \mathbf{1} & \mathbf{0} & \frac{1}{J} \mathrm{~N}_{1}^{\prime} \mathbf{1}
\end{array}\right]\left[\begin{array}{cc}
\mathbf{0} & \mathbf{0} \\
-\hat{\boldsymbol{\gamma}}_{i j} & \mathbf{0} \\
\mathbf{1} & \mathbf{0} \\
\mathbf{0} & \mathbf{1}
\end{array}\right]\left\{\begin{array}{c}
\boldsymbol{n}_{i j} \\
\boldsymbol{m}_{i j}
\end{array}\right\} J d \xi \\
& -\int_{-1}^{1}\left[\begin{array}{cccc}
\mathrm{N}_{0} \mathbf{1} & \mathbf{0} & \frac{1}{J} \mathrm{~N}_{0}^{\prime} \mathbf{1} & \mathbf{0} \\
\mathbf{0} & \mathrm{N}_{0} \mathbf{1} & \mathbf{0} & \frac{1}{J} \mathrm{~N}_{0}^{\prime} \mathbf{1}
\end{array}\right]\left[\begin{array}{cc}
\mathbf{0} & \mathbf{0} \\
-\hat{\boldsymbol{\gamma}}_{j k} & \mathbf{0} \\
\mathbf{1} & \mathbf{0} \\
\mathbf{0} & \mathbf{1}
\end{array}\right]\left\{\begin{array}{c}
\boldsymbol{n}_{j k} \\
\boldsymbol{m}_{j k}
\end{array}\right\} J d \xi
\end{aligned}
$$

\subsubsection{Deformations associated to rotations}

The next step is to calculate the change of orientation in each element. Following Crisfield and Jelenic [28, 29], a spherical interpolation of rotations throughout the element will be used for that purpose:

$$
\boldsymbol{\Lambda}^{h}(S)=\boldsymbol{\Lambda}_{i}\left(\boldsymbol{\Lambda}_{i}^{T} \boldsymbol{\Lambda}_{j}\right)^{S / L_{e}}
$$

For a 2-node element with nodes $i, j$ and actual length $L_{e}$, the local rotation $\boldsymbol{\Theta}_{i j}$, between $i$ and $j$ is computed as follows:

$$
\exp \hat{\boldsymbol{\Theta}}_{i j}=\boldsymbol{\Lambda}_{i}^{T} \boldsymbol{\Lambda}_{j}
$$

The rotation $\boldsymbol{\Lambda}_{r}$ at the midpoint of each element is used as element's reference rotation (Fig. 4); it is defined as:

$$
\boldsymbol{\Lambda}_{r}=\left.\boldsymbol{\Lambda}\right|_{\xi=0}=\boldsymbol{\Lambda}_{i} \exp \left(\frac{1}{2} \hat{\boldsymbol{\Theta}}_{i j}\right)
$$

Spherical interpolation is defined through the local rotation $\hat{\mathbf{\Psi}}_{e}=\frac{1}{2} \hat{\mathbf{\Theta}}_{i j}$ and the reference rotation, for $\xi \in[-1,1]$ as follows:

$$
\boldsymbol{\Lambda}(\xi)=\boldsymbol{\Lambda}_{r} \exp \left(\xi \hat{\mathbf{\Psi}}_{e}\right)
$$

Making use of the definition of curvature $\boldsymbol{K}$ :

$$
\begin{gathered}
\hat{\boldsymbol{K}}(\xi)=\boldsymbol{\Lambda}^{T} \frac{d}{d S} \boldsymbol{\Lambda}=\boldsymbol{\Lambda}^{T} \frac{d}{d \xi} \boldsymbol{\Lambda} \frac{d \xi}{d S} \\
=\exp \left(-\xi \hat{\boldsymbol{\Psi}}_{e}\right) \boldsymbol{\Lambda}_{r}^{T} \boldsymbol{\Lambda}_{r} \frac{d}{d \xi} \exp \left(\xi \hat{\boldsymbol{\Psi}}_{e}\right) \frac{2}{L_{e}} \\
=\mathbf{T}\left(\xi \hat{\mathbf{\Psi}}_{e}\right)^{T} \hat{\mathbf{\Psi}}_{e} \frac{2}{L_{e}}
\end{gathered}
$$


The operator $\mathbf{T}$ acts on $\hat{\boldsymbol{\theta}}$ as follows [24]:

$$
\mathbf{T}(\hat{\boldsymbol{\theta}})=\mathbf{1}+\frac{1-\cos \theta}{\theta^{2}} \hat{\boldsymbol{\theta}}+\frac{\theta-\sin \theta}{\theta^{3}} \hat{\boldsymbol{\theta}}^{2}
$$

where $\hat{\boldsymbol{\theta}}$ is a skew-symmetric matrix and $\theta$ is the modulus of the associated axial vector. Assuming that the deformation is constant in each element and evaluating it at $\xi=0$, the change of orientation $\boldsymbol{K}$ through the local rotation $\hat{\Psi}_{e}$ is calculated as:

$$
\boldsymbol{K}=\frac{2}{L_{0}} \boldsymbol{\Psi}_{e}
$$

and

$$
\boldsymbol{K}_{d}=\frac{2}{L_{0}} \boldsymbol{\Psi}_{e}-\boldsymbol{K}_{0}
$$

where $L_{0}$ is the prescribed target curve length for each discretization.

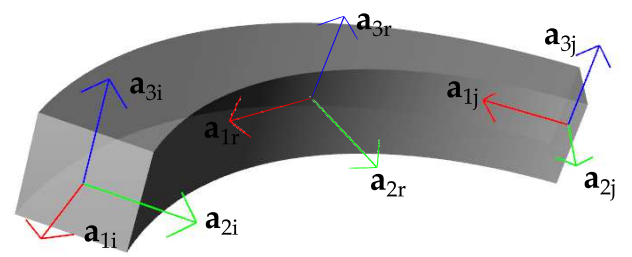

Figure 4: A typical element showing the nodal frames and the reference frame. $\Lambda_{r}$ is computed through spherical interpolation between $\Lambda_{i}$ and $\Lambda_{j}$.

\subsubsection{Deformations associated to translations}

In contrast to the change of orientation, the deformation associated to the traslations is computed in a straightforward way, only taking into account the spatial position of the nodes $i, j$ :

$$
\gamma=\frac{1}{L_{0}}\left(\boldsymbol{x}_{j}-\boldsymbol{x}_{i}\right)
$$

where $\boldsymbol{x}_{i}, \boldsymbol{x}_{j}$ are the position vectors of the nodes $i, j$ in spatial form. For rewriting it in material form, $\gamma$ will be pre-multiplicated by the transpose of the reference rotation matrix of the element:

$$
\boldsymbol{\Gamma}_{d}=\boldsymbol{\Lambda}_{r}^{T} \frac{1}{L_{0}}\left(\boldsymbol{x}_{j}-\boldsymbol{x}_{i}\right)-\{1,0,0\}^{T}
$$

\subsection{Numerical implementation}

The numerical solution of the dynamic equations is carried out via an one-step explicit algorithm based on Newmark's algorithm [30. According to the laws of motion Eqs. (19a) and (19b), the dynamic equations in spatial configuration for node $J$ at time $t$ are:

$$
\begin{aligned}
& \tilde{\boldsymbol{f}}_{n_{J}}^{t}=\mathbb{m}_{J} \boldsymbol{a}_{J}^{t}+\mathbb{C}_{J}\left(\dot{\boldsymbol{x}}_{J}^{t}+\boldsymbol{a}_{J}^{t} \frac{1}{2} \Delta t\right) \\
& \tilde{\boldsymbol{f}}_{m_{J}}^{t}=\AA_{\AA_{J}} \dot{\boldsymbol{\omega}}_{J}^{t}+\boldsymbol{\omega}_{J}^{t} \times \AA_{\AA_{J}} \boldsymbol{\omega}_{J}^{t}+\mathbb{C}_{J} \boldsymbol{\omega}_{J}^{t}
\end{aligned}
$$


Matrices $\mathfrak{m}_{J}$ and $i_{J}$ represent the fictitious nodal masses and inertias. Matrix $\mathbb{C}_{J}$ represents damping. The obtention of $m_{J}$ and $\stackrel{\circ}{J}_{J}$ is based on the relation between the mechanical properties of the rod and the timescale $\Delta t$ of the dynamic process; it ensures fast stability and avoids slow simulations. Based on reference [19], the following expressions in the material form are given:

$$
\begin{gathered}
M_{J}=\frac{\Delta t^{2}}{2}\left[\begin{array}{ccc}
\sum_{n e=1}^{k}\left(\frac{E A}{L_{0}}\right) & 0 & 0 \\
0 & \sum_{n e=1}^{k}\left(\frac{G A_{Q 2}}{L_{0}}\right) & 0 \\
0 & 0 & \sum_{n e=1}^{k}\left(\frac{G A_{Q 3}}{L_{0}}\right)
\end{array}\right] \\
\square_{J}=\beta \frac{\Delta t^{2}}{2}\left[\begin{array}{ccc}
\sum_{n e=1}^{k}\left(\frac{G J}{L_{0}}\right) & 0 & 0 \\
0 & \sum_{n e=1}^{k}\left(\frac{E I_{2}}{L_{0}}\right) & 0 \\
0 & 0 & \sum_{n e=1}^{k}\left(\frac{E I_{3}}{L_{0}}\right)
\end{array}\right]
\end{gathered}
$$

Moreover, an additional coefficient $\beta$ can be introduced to increase the damping related to the bending effect and prevent non-convergence. Pulling back material variables, the spatial description of the fictitious nodal properties is:

$$
\begin{gathered}
\mathrm{m}_{J}=\boldsymbol{\Lambda}_{J} \mathbb{M}_{J} \boldsymbol{\Lambda}_{J}^{T} \\
\stackrel{\circ}{\Xi}_{J}=\boldsymbol{\Lambda}_{J} \square_{J} \boldsymbol{\Lambda}_{J}^{T}
\end{gathered}
$$

Manipulating equations $37 \mathrm{a}$ and $37 \mathrm{~b}$, it is possible to assess the translational $\boldsymbol{a}_{J}^{t}$ and rotational $\dot{\boldsymbol{\omega}}_{J}^{t}$ accelerations of the nodes $\boldsymbol{x}_{J}$ at time $t$ :

$$
\begin{aligned}
& \boldsymbol{a}_{J}^{t}=\left(\mathrm{m}_{J}+\mathbb{C}_{J} \frac{1}{2} \Delta t\right)^{-1}\left(\tilde{\boldsymbol{f}}_{n_{J}}^{t}-\mathbb{C}_{J} \dot{\boldsymbol{x}}_{J}^{t}\right)
\end{aligned}
$$

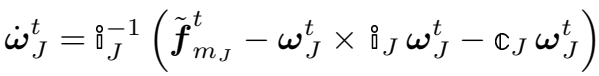

With the obtained translational accelerations, positions $\boldsymbol{x}_{J}^{t+\Delta t}$ and translational velocities $\dot{\boldsymbol{x}}_{J}^{t+\Delta t}$ are updated as:

$$
\begin{aligned}
& \boldsymbol{x}_{J}^{t+\Delta t}=\boldsymbol{x}_{J}^{t}+\dot{\boldsymbol{x}}_{J}^{t} \Delta t+\frac{1}{2} \boldsymbol{a}_{J}^{t} \Delta t^{2} \\
& \dot{\boldsymbol{x}}_{J}^{t+\Delta t}=C_{1} \dot{\boldsymbol{x}}_{J}^{t}+C_{2} \boldsymbol{a}_{J}^{t} \Delta t
\end{aligned}
$$

where $C_{1}$ and $C_{2} \in[0,1]$ are the terms of viscous damping used to avoid large oscillations in the free nodes, which can produce numerical instabilities [10]. Angular velocities $\boldsymbol{\omega}_{J}^{t}$ and $\boldsymbol{\omega}_{J}^{t+\Delta t}$ are not additive because they belong to different frames. To overcome this problem, a strategy based on the one proposed by Ibrahimbegovic [31] to represent the change of orientation of sections is used:

$$
\boldsymbol{\omega}_{J}^{t+\Delta t}=\mathbf{T}\left(\Delta \hat{\boldsymbol{\omega}}_{J}^{t}\right) \Delta \dot{\boldsymbol{\omega}}_{J}^{t}+\exp \left(\Delta \hat{\boldsymbol{\omega}}_{J}^{t}\right) \boldsymbol{\omega}_{J}^{t}
$$

with:

$$
\begin{aligned}
\Delta \boldsymbol{\omega}_{J}^{t} & =\boldsymbol{\omega}_{J}^{t} \Delta t \\
\Delta \dot{\boldsymbol{\omega}}_{J}^{t} & =\dot{\boldsymbol{\omega}}_{J}^{t} \Delta t
\end{aligned}
$$

The operator $\mathbf{T}$ has been defined in Eq. 32 and $\exp (\Delta \hat{\boldsymbol{\omega}})$ can be calculated using Rodrigues's formula:

$$
\exp (\Delta \hat{\boldsymbol{\omega}})=\mathbf{1}+\frac{\sin \Delta \omega}{\Delta \omega} \Delta \hat{\boldsymbol{\omega}}+\frac{1-\cos \Delta \omega}{\Delta \omega^{2}} \Delta \hat{\boldsymbol{\omega}}^{2}
$$


where $\Delta \boldsymbol{\omega}$ is the incremental nodal rotation vector, $\Delta \omega$ is its modulus and $\Delta \hat{\boldsymbol{\omega}}$ is the antisymmetric matrix associated to $\Delta \omega$. The update of the nodal rotation is therefore:

$$
\boldsymbol{\Lambda}_{J}^{t+\Delta t}=\exp \left(\Delta \hat{\boldsymbol{\omega}}_{J}^{t}\right) \boldsymbol{\Lambda}_{J}^{t}
$$

\subsubsection{Criterium for convergency}

The dynamic relaxation algorithm must iterate up to reach the criterium for convergency, based on the evaluation of translational and rotational kinetic energies:

$$
\begin{aligned}
\mathcal{K}_{t_{J}} & =\frac{1}{2} \dot{\boldsymbol{x}}_{J}^{T} \mathrm{~m}_{J} \dot{\boldsymbol{x}}_{J} \\
\mathcal{K}_{r_{J}} & =\frac{1}{2} \boldsymbol{\omega}_{J}^{T \mathrm{~g}_{J}} \boldsymbol{\omega}_{J}
\end{aligned}
$$

$\mathcal{K}_{t_{J}}$ and $\mathcal{K}_{r_{J}}$ are the kinetic energies corresponding to node $J$. When all energies reach a prescribed limit value, the solution is regarded as a static equilibrium configuration and the algorithm stop.

\subsubsection{Boundary conditions}

The choice of the geometrically exact rod model allows to define kinematic constraints in a straightforward way, since neither ficticious extensions of the rod nor additional constraints [13, are required. The authors understand the concept of form-finding as a 'design-oriented' procedure [13], in which equilibrium is found after defining a target curve length and the corresponding kinematic constraints. In other words, the proposed form-finding method is based on geometrical restrictions. The algorithm has been designed to deal with two possibilities: either positions and rotations at the beam ends are fixed, or positions are fixed and rotations are free.

\section{Numerical tests}

\subsection{The elastica and the circular beam}

The first example is a beam clamped at both ends; the distance between them is $10 \mathrm{~m}$. The mechanical properties of the beam are shown in table 1 and correspond to a rectangular crosssection with $2 \mathrm{~cm}$ width and $0.5 \mathrm{~cm}$ depth; only $I_{2}$ and $A_{Q 3}$ are relevant because the weak axis is assumed to be the bending axis. The modulus of elasticity is $E=10^{4} \mathrm{MPa}$, the relation $E / G$ is 2.5 and the time increment is $\Delta t=0.0001 \mathrm{~s}$. The additional imposed kinematic boundary condition is the cross-section orientation at beam ends where the centreline of the curve forms an angle of $30^{\circ}$ with the $x$-axis. Non-fixed nodal frames are initially defined by spherical interpolation.

Among the wide range of possible curves with different prescribed target lengths $L_{0}$ that fulfil the established constraints, the elastica is defined by $L_{0}=10.725 \mathrm{~m}$ and the circular beam with radius $R=10 \mathrm{~m}$ is defined by $L_{0}=10.47 \mathrm{~m}$. Fig. 5 shows the equilibrium geometries in both cases using 10 elements. The errors registered for the $y$-coordinate at the midspan of the elastica and the circular beam are $0.05 \%$ and $0.04 \%$ respectively. Another straightforward way of validating these models is to assess curvatures $\kappa$ of the beams. Fig. 6 shows an excellent correlation between numerical and analytical curvatures in both tests. The circular beam has a constant curvature of value $\kappa=-0.1 \mathrm{~m}^{-1}$ defined by the relation $1 / R$ whereas the curvature of the elastica can be calculated analitically [32] 6]. It is worth remarking that curvatures are computed at the Gauss's point of each element (at the middle of the element), not at nodes, as outlined above. For this reason, in the elastica, curvature values at elements 1 and 10 are very low but non-zero, since they do not correspond with the beam end nodes. Fig. 7 shows how the curve lengths grow to the target value $L_{0}$ during the form-finding process. 


\begin{tabular}{cccccc}
\hline $\begin{array}{c}A \\
\left(\mathrm{~cm}^{2}\right)\end{array}$ & $\begin{array}{c}A_{Q 2} \\
\left(\mathrm{~cm}^{2}\right)\end{array}$ & $\begin{array}{c}A_{Q 3} \\
\left(\mathrm{~cm}^{2}\right)\end{array}$ & $\begin{array}{c}J \\
\left(\mathrm{~cm}^{4}\right)\end{array}$ & $\begin{array}{c}I_{2} \\
\left(\mathrm{~cm}^{4}\right)\end{array}$ & $\begin{array}{c}I_{3} \\
\left(\mathrm{~cm}^{4}\right)\end{array}$ \\
\hline 1 & 0.8333 & 0.8333 & 0.4621 & 0.0208 & 0.3333 \\
\hline
\end{tabular}

Table 1: Section properties of the elastica and the circular beam.

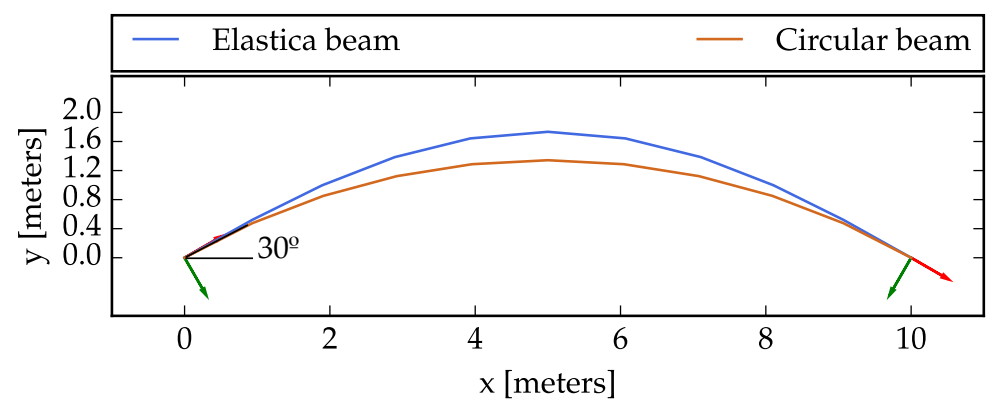

Figure 5: Form-finding of the elastica and the circular beam defined by the same local reference frames at the beam ends.

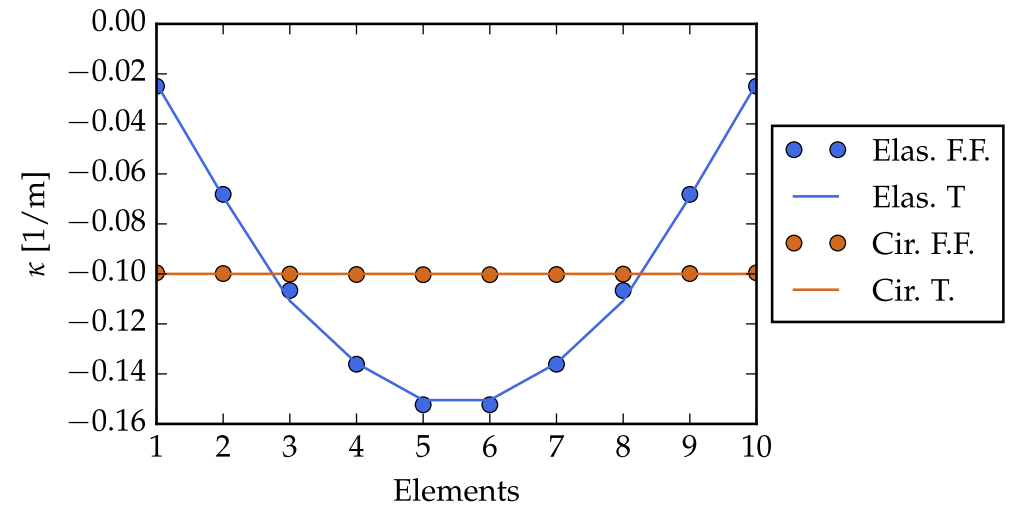

Figure 6: Curvatures for the elastica (Elas.) in blue and curvatures for the circular beam (Cir.) in brown. Form-finding (F.F.) and theoretical solutions (T.). 


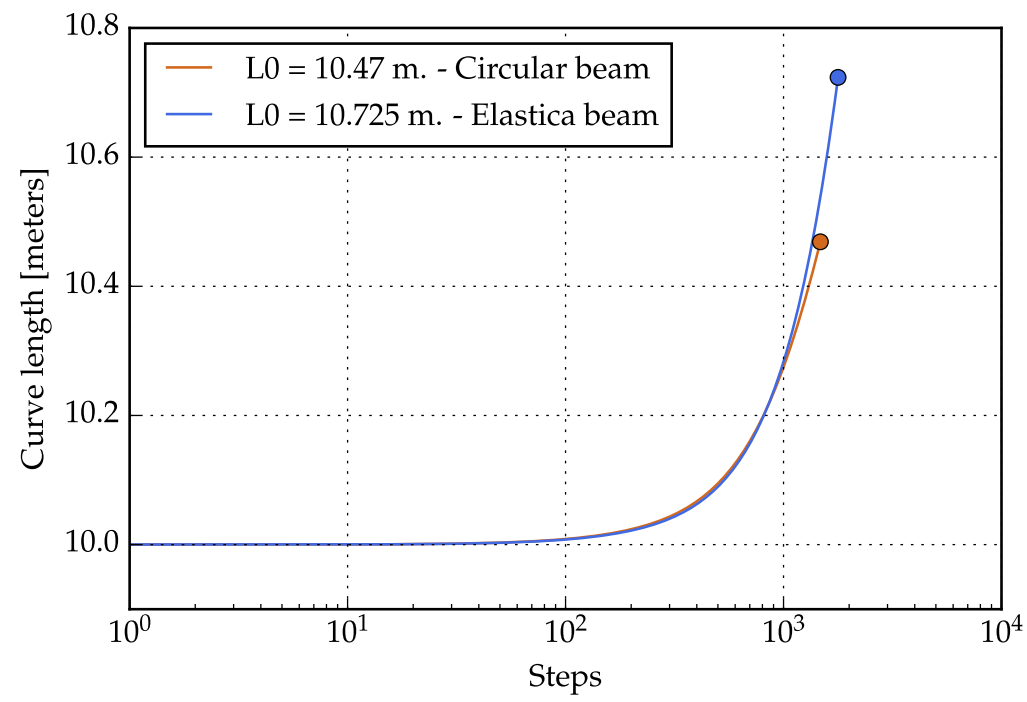

Figure 7: Evolution of the curve lengths for the elastica and the circular beam during the form-finding process.

In parallel, Fig. (8) shows the form-finding process at different stages, indicating both the number of the step and the actual curve length. Finally, this example allows to show that the method also works when the orientations of the beam ends are not restricted: allowing free rotations at both ends and establishing $L_{0}=10.725 \mathrm{~m}$ (length of the elastica), the start/final angle of the curve in the equilibrium state is $30.05^{\circ}$, practically the exact solution. In other words, allowing free rotations at both ends of the rod, the obtained curve is the elastica associated to the prescribed target length $L_{0}$.

\subsection{Bathe and Bolourchi cantilever beam}

The second example is well-known in the finite-element literature [33. The original problem is a circular cantilever beam clamped at the origin and subject to a force at its free node (Fig. 9). The units of the original problem have been kept for consistency. Initially, the curved beam is contained within the plane $z=0$, the curve has a radius of 100 in comprising an angle of $45^{\circ}$ and the material and section properties are shown in the table 2. Bathe and Simo solved this problem taking into account a transverse force of $600 \mathrm{lb}$ at the free end of the beam using 8 two-node elements in their models.

Knowing the solution by means of a implicit numerical tool based on Simo's model [17, it is possible to transform a load analysis example into a 'design oriented' form-finding problem by imposing the target kinematic constraints at both beam ends. Fig. (10) shows the evolution of the translational and rotational kinetic energies in the form-finding process. Moreover, three captures of the geometry at different stages show how initially the beam is practically straight (a) whereas and at the end of the process, when the kinetic energies are close to zero, the equilibrium geometry is reached according to the imposed orientations at both beam ends (c). Fig. (11) shows the high correspondency between the nodal coordinates of the obtained solution by the implicit and the form-finding method using 8 two-node elements. 


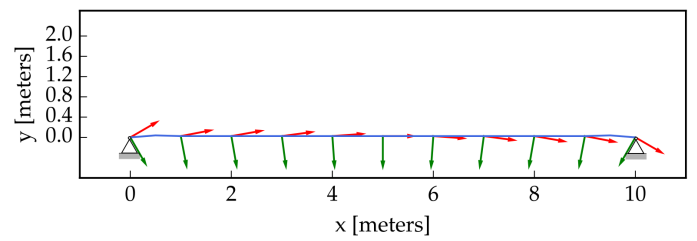

(a) Step 0; $L=10 \mathrm{~m}$.

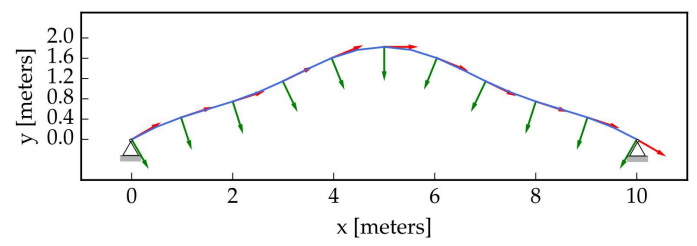

(c) Step 890; $L=10.69 \mathrm{~m}$.

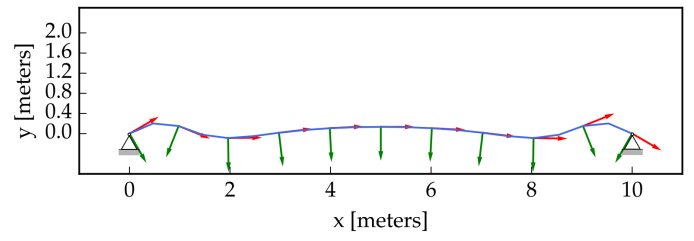

(b) Step 100; $L=10.17 \mathrm{~m}$.

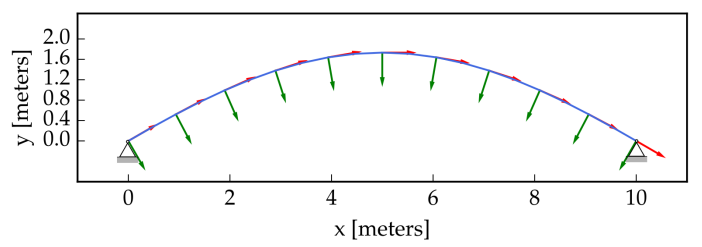

(d) Step 1780; $L=10.725 \mathrm{~m}$.

Figure 8: Evolution of the form-finding process of a elastica curve and its length $(L)$ at different stages.

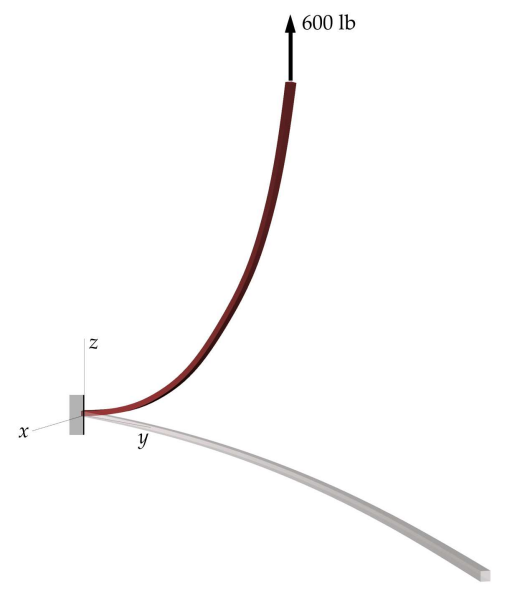

Figure 9: Original problem of the Bathe and Bolourchi cantilever beam.

\begin{tabular}{cccccccc}
\hline $\begin{array}{c}A \\
\left(i n^{2}\right)\end{array}$ & $\begin{array}{c}A_{Q 2} \\
\left(i n^{2}\right)\end{array}$ & $\begin{array}{c}A_{Q 3} \\
\left(i n^{2}\right)\end{array}$ & $\begin{array}{c}J \\
\left(i n^{4}\right)\end{array}$ & $\begin{array}{c}I_{2} \\
\left(i n^{4}\right)\end{array}$ & $\begin{array}{c}I_{3} \\
\left(i n^{4}\right)\end{array}$ & $\begin{array}{c}E \\
\left(l b / i n^{2}\right)\end{array}$ & $E / G$ \\
\hline 1 & 1 & 1 & $1 / 6$ & $1 / 12$ & $1 / 12$ & $10^{7}$ & 2 \\
\hline
\end{tabular}

Table 2: Material and section properties of the Bathe and Bolourchi cantilever beam. 


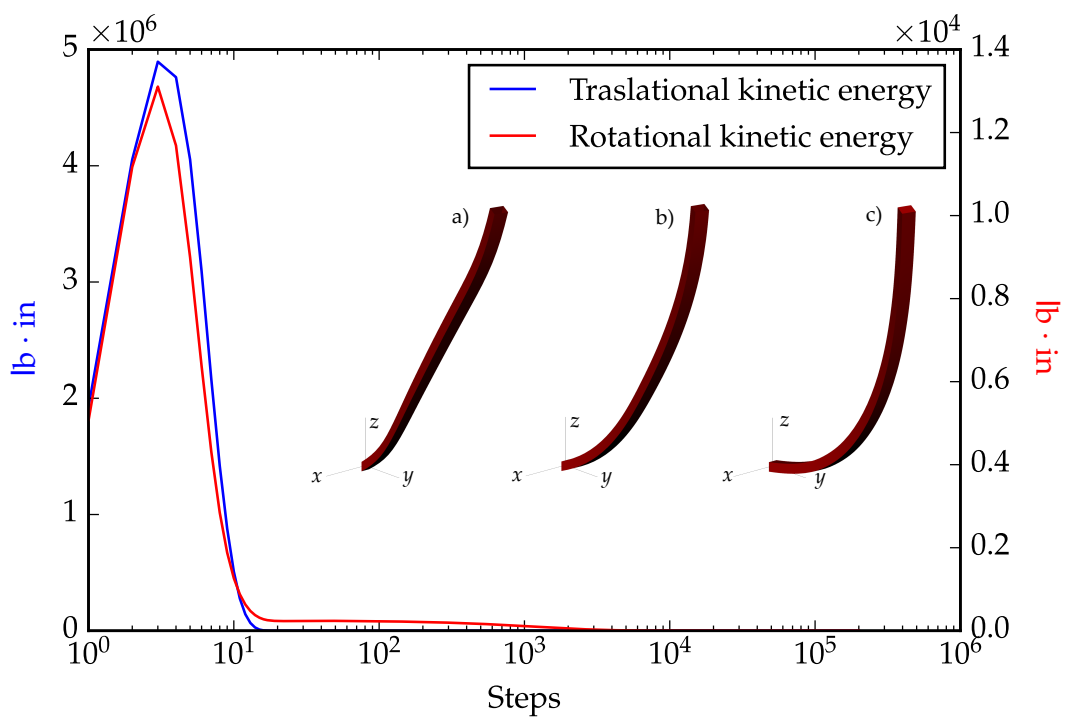

Figure 10: Evolution of the kinetic energies for the Bathe and Bolourchi cantilever beam. Figure a) shows the initial geometry. Figure b) corresponds to the geometry at step 100. Figure c) shows the final equilibrium geometry.

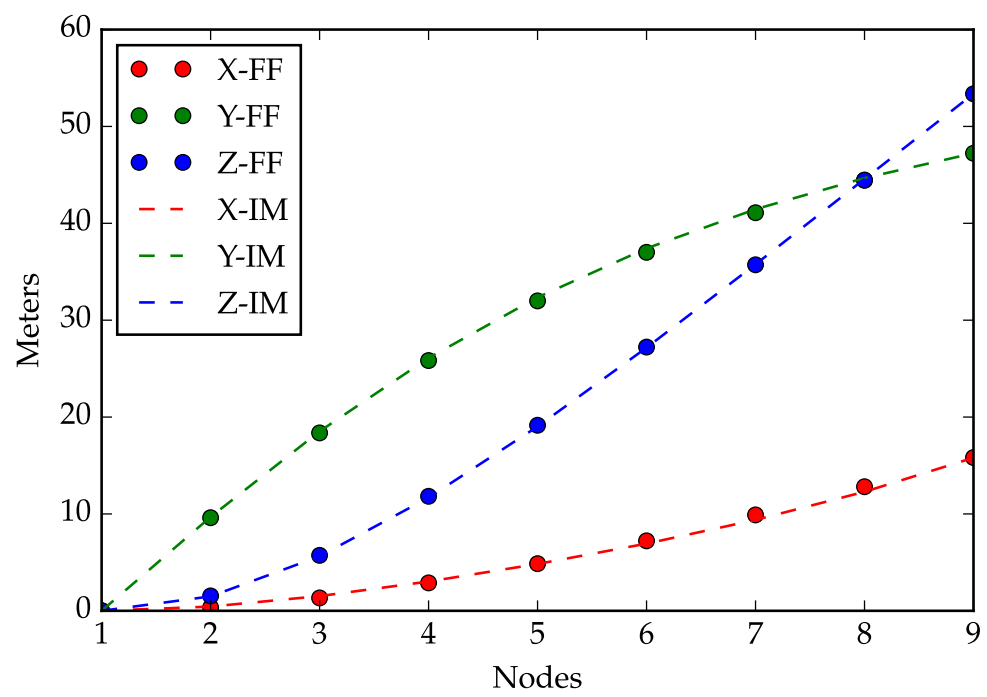

Figure 11: Coordinates $X-Y-Z$ using the form-finding method (F.F.) and the implicit numerical solution based on Simo's theory (I.M.) for the Bathe and Bolourchi cantilever beam. 


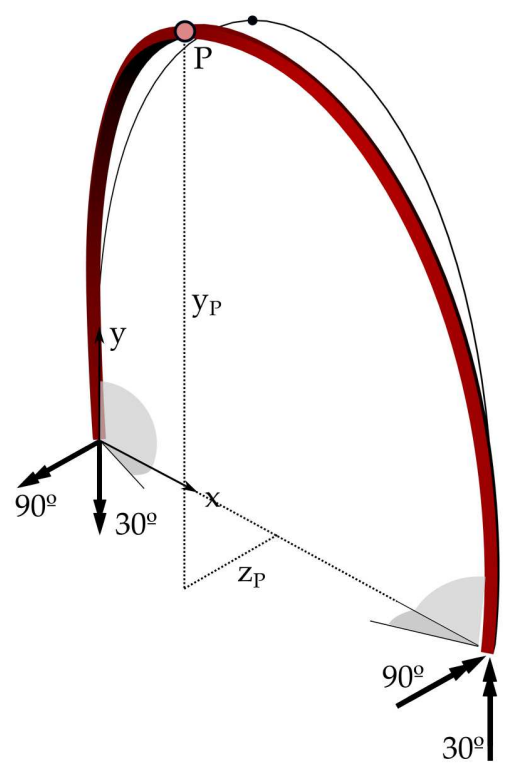

Figure 12: An initially straight rod is bent up to reach vertical tangents at supports and then twisted by rotating $30^{\circ}$ its ends.

\begin{tabular}{cccccc}
\hline $\begin{array}{c}A \\
\left(\mathrm{~cm}^{2}\right)\end{array}$ & $\begin{array}{c}A_{Q 2} \\
\left(\mathrm{~cm}^{2}\right)\end{array}$ & $\begin{array}{c}A_{Q 3} \\
\left(\mathrm{~cm}^{2}\right)\end{array}$ & $\begin{array}{c}J \\
\left(\mathrm{~cm}^{4}\right)\end{array}$ & $\begin{array}{c}I_{2} \\
\left(\mathrm{~cm}^{4}\right)\end{array}$ & $\begin{array}{c}I_{3} \\
\left(\mathrm{~cm}^{4}\right)\end{array}$ \\
\hline 2400 & 2000 & 2000 & 91.147 & 32 & 72 \\
\hline
\end{tabular}

Table 3: Section properties for the case of bending and torsion effects.

\subsection{Combined bending and torsion}

This example simulates the behaviour of a $10 \mathrm{~m}$ bent beam when its ends are clamped at a distance of $4.56 \mathrm{~m}$ and twisted $30^{\circ}$. For that purpose, the kinematic constraints at both ends are specified by setting the orientation of each cross-section through the definition of the corresponding reference frames (see Fig, 12). By means of two elemental rotations, firstly, the tangent vectors are rotated an angle of $90^{\circ}$ for simulating bending effects. When convergence is reached, a second form-finding step is carried out rotating the end frames about the tangent vectors an angle of $30^{\circ}$ to evaluate twisting effects. Cross-section properties are shown in the table 3 . The modulus of elasticity is $E=10^{4} \mathrm{MPa}$, the relation $E / G$ is 2.5 and the selected time interval is $\Delta t=0.0001 \mathrm{~s}$. To assess the reliability of the method, the form-finding solution is compared with the obtained results by the same numerical implicit tool used in the example 2, based on Simo's procedure. Both models are discretised into an increasing number of elements (from 8 to 20) and ratios between coordinates corresponding to the node ' $\mathrm{P}$ ' and the beam length are shown in Table 4 . The algorithm stops when both translational and rotational kinetic energies are under $10^{-7} \mathrm{kN} \cdot \mathrm{m}$, after approximately 100.000 steps. As it can be seen in table 3, the form-finding method provides reliable results; the error diminishes as the number of elements increases. 


\begin{tabular}{lcccc}
\hline \multirow{2}{*}{ Num. of elements } & \multicolumn{2}{c}{$\Delta y_{p} / L$} & \multicolumn{2}{c}{$\Delta z_{p} / L$} \\
\cline { 2 - 5 } & I.M. & F.F. & I.M. & F.F. \\
\hline 8 & 0.3796 & 0.3876 & -0.0357 & -0.0390 \\
16 & 0.3795 & 0.3862 & -0.0343 & -0.0365 \\
20 & 0.3795 & 0.3861 & -0.0341 & -0.0355 \\
\hline
\end{tabular}

Table 4: Vertical and horizontal relative deflection for the bent and twisted beam taking into account different number of elements in both form-finding method (F.F.) and implicit method based on Simo's theory (I.M.).

\begin{tabular}{cccccc}
\hline $\begin{array}{c}A \\
\left(\mathrm{~cm}^{2}\right)\end{array}$ & $\begin{array}{c}A_{Q 2} \\
\left(\mathrm{~cm}^{2}\right)\end{array}$ & $\begin{array}{c}A_{Q 3} \\
\left(\mathrm{~cm}^{2}\right)\end{array}$ & $\begin{array}{c}J \\
\left(\mathrm{~cm}^{4}\right)\end{array}$ & $\begin{array}{c}I_{2} \\
\left(\mathrm{~cm}^{4}\right)\end{array}$ & $\begin{array}{c}I_{3} \\
\left(\mathrm{~cm}^{4}\right)\end{array}$ \\
\hline 1 & 0.5 & 0.5 & 0.0137 & 2.0416 & 0.0416 \\
\hline
\end{tabular}

Table 5: Section properties of the beam used in the shear deformability test.

\subsection{Shear deformability test}

This example is intended to study a case where shear effects are not negligible and can be simulated by the form-finding method presented in this work. The benchmark for this shear deformability test is the elastica of the first example with the same prescribed target length $\left(L_{0}=10.725 \mathrm{~m}\right)$ and distance between beam ends $(10 \mathrm{~m})$. The modulus of elasticity is $E=10^{4} \mathrm{MPa}$, the relation $E / G$ is 2.5 and the selected time interval is $\Delta t=0.0001 \mathrm{~s}$. The mechanical properties of the beam under study are shown in table 5 . Such properties have been chosen to get a lower ratio between the shear stiffness and the bending stiffness than in the case of the elastica, being both cross-sectional areas equivalent. The relation between shear factors $\alpha=12 E I_{2} /\left(G A_{Q 3} L_{0}^{2}\right)$ in both cases is:

$$
\alpha_{\text {shear test }} / \alpha_{\text {elastica }}=163.5
$$

Fig. 13 shows the transverse shear deformations corresponding to both, the elastica case (blue) and the shear deformable case (brown). As expected, shear deformations are much larger than in the elastica case. Therefore, this example highlights the ability of the proposed form-finding method to reproduce shear deformable cases. 


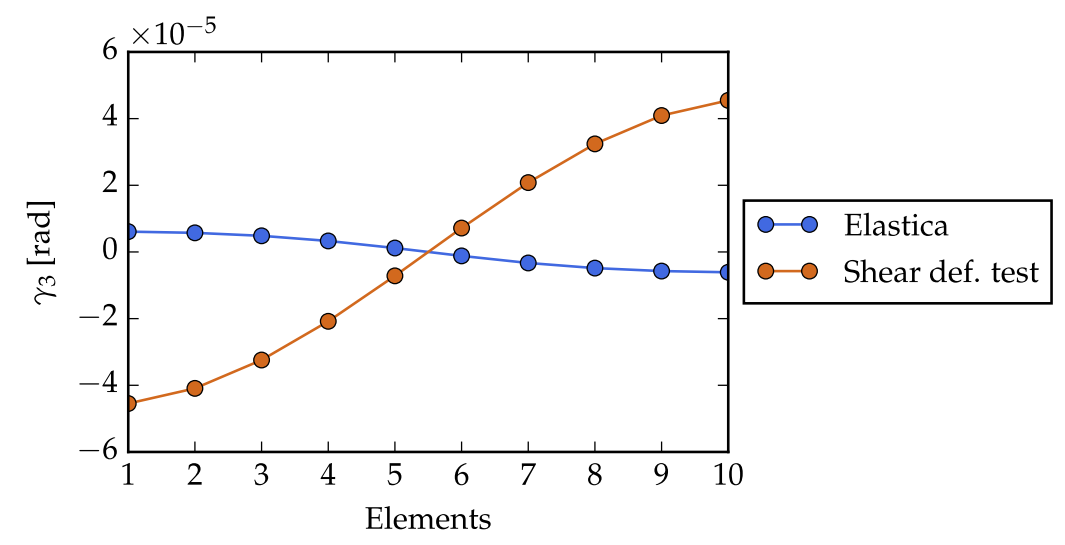

Figure 13: Shear deformations for the elastica and a beam where transverse shear is not negligible.

\section{Concluding remarks}

A form-finding method for bending-active structures implementing the so-called geometrically exact rod model into a dynamic relaxation procedure has been developed. The geometrically exact beam model was designed by Reissner, Simo and other authors to handle mechanical problems of slender rods undergoing large displacements and rotations, therefore it has been selected as underlying mechanical model in our implementation. The use of 6 DoFs per node to update translations and rotations provides a complete description of the kinematics and allows for a straightforward definition of rotational boundary conditions. Key points in the specialization of Reissner-Simo's model to the dynamic relaxation procedure have been: the derivation of element end forces from the internal virtual work equation (sect. 2.3.2); the derivation of the expression of the change of orientation in the element (sect. 2.3.3); the definition of the numerical step using Newmark's algorithm and the full expression of the inertial torques (Eq. 37b); and the update of angular velocities considering that nodal frames are changing (Eq. 42). Dynamic relaxation provides realiable results in terms of accuracy and computation time, as well as the possibility of monitoring the numerical process through different parameters: curve length, kinetic energies, number of steps or residual forces.

In contrast to the work of other authors, in the studied examples the form-finding process is driven by kinematic constraints, defining restrictions at end nodes and using the beam length as a design parameter. We showed that different lengths or end restrictions lead to meaningful solutions of the form-finding problem. The numerical examples also illustrated the ability and accuracy of the method to reproduce the configuration of active members starting from an initial geometry far from the equilibrium solution.

\section{Acknowledgements}

The financial support from the Spanish Ministry of Economy and Competitiveness through grant BIA2015-69330-P (MINECO) is gratefully acknowledged. 


\section{References}

[1] E. Happold and W. Liddell. Timber Lattice Roof for the Mannheim Bundesgartenschau. Structural Engineer, 53(3):99-135, 1975.

[2] Lionel Du Peloux, Frédéric Tayeb, Jean-François Caron, and Olivier Baverel. The Ephemeral Cathedral of Créteil : a $350 \mathrm{~m} 2$ lightweight gridshell structure made of 2 kilometers of GFRP tubes. In Proceedings of CIGOS 2015, Cachan, 2015.

[3] Julian Lienhard, Holger Alpermann, Christoph Gengnagel, and Jan Knippers. Active Bending, A Review on Structures where Bending is used as a Self-Formation Process. International Journal of Space Structures, 28(3-4):187-196, sep 2013.

[4] J. Lienhard. Bending-active structures. Form-finding strategies using elastic deformation in static and kinematic systems and the structural potentials therein. PhD thesis, Stuttgart, 2014.

[5] J. Lienhard, R. La Magna, and J. Knippers. Form-finding bending-active structures with temporary ultra-elastic contraction elements. WIT Transactions on the Built Environment, 136:107-116, 2014.

[6] Carlos Lázaro, Salvador Monleón, and José Casanova. Can the force density method be extended for active bending structures? In Proceedings of the IASS Annual Symposium 2015, Amsterdam, 2015.

[7] S.M.L Adriaenssens and M.R Barnes. Tensegrity spline beam and grid shell structures. Engineering Structures, 23(1):29-36, 2001.

[8] C. Douthe, O. Baverel, and J.F. Caron. Form-finding of a grid shell in composite material. Journal of the International Association for Shell and Spatial Structures, 47:53-62, 2006.

[9] Michael R. Barnes, Sigrid Adriaenssens, and Meghan Krupka. A novel torsion/bending element for dynamic relaxation modeling. Computers \& Structures, 119:60-67, 2013.

[10] B. D'Amico, A. Kermani, and H. Zhang. Form finding and structural analysis of actively bent timber grid shells. Engineering Structures, 81:195-207, 2014.

[11] Lionel Du Peloux, Frédéric Tayeb, Baptiste Lefevre, Olivier Baverel, and Jean-Francois Caron. Formulation of a 4-DoF torsion/bending element for the formfinding of elastic gridshells. In Proceedings of the IASS Symposium 2015, pages 1-15, Amsterdam, 2015.

[12] Gennaro Senatore and Daniel Piker. Interactive real-time physics: An intuitive approach to form-finding and structural analysis for design and education. Computer-Aided Design, 61:3241, 2015.

[13] B. D'Amico, H. Zhang, and A. Kermani. A finite-difference formulation of elastic rod for the design of actively bent structures. Engineering Structures, 117:518-527, 2016.

[14] Baptiste Lefevre, Frédéric Tayeb, Lionel du Peloux, and Jean-François Caron. A 4-degree-offreedom Kirchhoff beam model for the modeling of bending-torsion couplings in active-bending structures. International Journal of Space Structures, 32(2):69-83, jun 2017.

[15] G. Kirchhoff. Über das Gleichgewicht und die Bewegung eines unendlich dünnen elastischen Stabes. Journal für reine und angewandte Mathematik, 56:285-313, 1859.

[16] E. Reissner. On one-dimensional finite-strain beam theory: The plane problem. Stud. Appl. Math., 52:87-95, 1973. 
[17] J.C. Simo. A finite strain beam formulation. The three-dimensional dynamic problem. Part I. Computer Methods in Applied Mechanics and Engineering, 49(1):55-70, 1985.

[18] J.C. Simo and L. Vu-Quoc. A Geometrically-exact rod model incorporating shear and torsionwarping deformation. International Journal of Solids and Structures, 27(3):371-393, 1991.

[19] M. R. Barnes. Form finding and analysys of tension structures by dynamic relaxation. International Journal of Space Structures, 14:89-104, 1999.

[20] E L Starostin and G H M Van Der Heijden. The shape of a Möbius strip. Nature Materials, 6:563-567, 2007.

[21] D. Wakefield. Dynamic relaxation analysis of pretensioned networks supported by compression arches. PhD thesis, London, 1980.

[22] S. Adriaenssens. Stressed spline structures. PhD thesis, Bath, 2000.

[23] Miklós Bergou, Max Wardetzky, Stephen Robinson, Basile Audoly, and Eitan Grinspun. Discrete elastic rods. ACM Transactions on Graphics, 27:1, 2008.

[24] M. Ritto-Corrêa and D. Camotim. On the differentiation of the Rodrigues formula and its significance for the vector-like parameterization of Reissner-Simo beam theory. International Journal for Numerical Methods in Engineering, 55(9):1005-1032, nov 2002.

[25] Carlos Lázaro, Salvador Monleón, Juan Bessini, and José Casanova. A review on geometrically exact models for very flexible rods. In Proceedings of the IASS Annual Symposium 2016, Tokyo, 2016.

[26] A. Day. An introduction to dynamic relaxation. Engineer 1965, 331(219):218-221, may 1965.

[27] J. R. H. Otter, A. C. Casell, R. E. Hobbs, and Poisson. Dynamic relaxation. Proceedings of the Institution of Civil Engineers, 35(4):633-656, dec 1966.

[28] M. A. Crisfield and G. Jelenić. Objectivity of strain measures in the geometrically exact threedimensional beam theory and its finite-element implementation. Proceedings of the Royal Society A: Mathematical, Physical and Engineering Sciences, 455(1983):1125-1147, mar 1999.

[29] G. Jelenić and M.A. Crisfield. Geometrically exact 3D beam theory: implementation of a straininvariant finite element for statics and dynamics. Computer Methods in Applied Mechanics and Engineering, 171(1-2):141-171, mar 1999.

[30] O. C. Zienkiewicz and R. L. Taylor. Finite Element Method: Volume 2, Solid Mechanics. Butterworth-Heinemann, Boston, 2000.

[31] Adnan Ibrahimbegović. On finite element implementation of geometrically nonlinear Reissner's beam theory: three-dimensional curved beam elements. Computer Methods in Applied Mechanics and Engineering, 122(1-2):11-26, apr 1995.

[32] AEH. Love. A Treatise on the Mathematical Theory of Elasticity. Dover, New York, 1944.

[33] K.J. Bathe and Bolourchi S. Large displacement analysis of three-dimensional beam structures. International Journal for Numerical Methods in Engineering, 14(7):961-986, 1979. 